\title{
Introduction to the Minitrack on Explainable Artificial Intelligence (XAI)
}

\author{
Christian Meske \\ Freie Universität Berlin \\ christian.meske@,fu-berlin.de
}

\author{
Babak Abedin \\ Macquarie University \\ babak.abedin@mq.edu.au
}

\author{
Fethi Rabhi \\ University of N. S. Wales \\ f.rabhi@unsw.edu.au
}

\author{
Iris Junglas \\ College of Charleson \\ junglasia@,cofc.edu
}

\begin{abstract}
The use of Artificial Intelligence (AI) in the context of decision analytics and service science has received significant attention in academia and practice alike. Yet, much of the current efforts have focused on advancing underlying algorithms and not on decreasing the complexity of AI systems. AI systems are still "black boxes" that are difficult to comprehend-not only for developers, but particularly for users and decisionmakers.
\end{abstract}

\section{Introduction}

The use of Artificial Intelligence (AI) in the context of decision analytics and service science has received significant attention in academia and practice alike. Yet, much of the current efforts have focused on advancing underlying algorithms and not on decreasing the complexity of AI systems. AI systems are still "black boxes" that are difficult to comprehend-not only for developers, but particularly for users and decisionmakers.

This is where research on Explainable Artificial Intelligence (XAI) becomes relevant [1]. Also referred to as "transparent," "interpretable," or "understandable AI," XAI aims to "produce explainable models, while maintaining a high level of learning performance (prediction accuracy); and enable human users to understand, appropriately, trust, and effectively manage the emerging generation of artificially intelligent partners" [2]. XAI hence refers to "the movement, initiatives, and efforts made in response to AI transparency and trust concerns, more than to a formal technical concept" [3, p. 5140)].

With a focus on decision support, this minitrack explores and extends research on how to establish explainability of intelligent black box systemsmachine learning-based or not. We received many highquality submissions, of which we were able to accept four.

\section{Papers}

In the first paper "Capturing Users' Reality: A Novel Approach to Generate Coherent Counterfactual Explanations", the authors design a novel optimizationbased approach to generate coherent counterfactual explanations. This approach is applicable to numerical, categorical as well as mixed data, and is demonstrated in a realistic setting to assess its efficacy in a humangrounded evaluation. The results suggest that the approach produces coherent explanations that are suitable to comprehend the factual situation.

The second paper, "AI-Assisted and Explainable Hate Speech Detection for Social Media Moderators" applies a Design Science Research approach in order to develop and evaluate design knowledge, through which humans are integrated in the process of detecting and evaluating hate speech. The resulting artifact provides explanative information on why the deep learning model predicted whether a text contains hate speech. The evaluation shows that the instantiated design principles in the form of a dashboard is perceived as valuable and that XAI features increase the perception of the artifact's usefulness and trustworthiness.

In the third paper, "What Your Radiologist Might be Missing: Using Machine Learning to Identify Mislabeled Instances of X-ray Images", the authors propose a two-staged pipeline for the automatic detection of potentially mislabeled instances in a large medical data set. Their results show that the pipeline successfully detects mislabeled instances of Cardiomegaly in the data set, contributing to ongoing efforts regarding data quality in machine learning.

The fourth and last paper, "Reviewing the Need for Explainable Artificial Intelligence (xAI)" is based on a literature review. With a systematic review of XAI literature the authors identify four thematic debates central to how XAI addresses the black-box problem. Based on the literature analysis, the authors synthesize the findings into a research agenda that can be used as a basis to extend the body of knowledge in XAI research. 


\section{References}

[1] Meske, C., Bunde, E., Schneider, J. and Gersch, M. (2021): Explainable Artificial Intelligence: Objectives, Stakeholders and Future Research Opportunities. Information Systems Management. Forthcoming.

[2] Gunning, D. (2017). Explainable Artificial Intelligence (XAI). DARPA Program Update November 2017. https://www.darpa.mil/attachments/XAIProgramUpdate.pdf

[3] Adadi, A., and Berrada, M. (2018). Peeking Inside the Black-Box: A Survey on Explainable Artificial Intelligence (XAI). IEEE Access, 6, 52138-52160. 